\title{
$S=+1$ pentaquarks in QCD sum rules
}

\author{
Philipp Gubler* \\ Department of Physics, H-27, Tokyo Institute of Technology, Meguro, Tokyo 152-8551, Japan \\ E-mail: phileth.phys.titech.ac.jp
}

\section{Daisuke Jido}

Yukawa Institute for Theoretical Physics, Kyoto University, Kyoto 606-8502, Japan

\section{Toru Kojo}

RBRC, Brookhaven National Laboratory, Upton, NY 11973-5000, USA

\section{Tetsuo Nishikawa}

Faculty of Health Science, Ryotokuji University, Urayasu, Chiba, 279-8567, Japan

\section{Makoto Oka}

Department of Physics, H-27, Tokyo Institute of Technology, Meguro, Tokyo 152-8551, Japan

\begin{abstract}
Pentaquark states with strangeness $S=+1$ and $I J^{\pi}=0 \frac{1}{2}^{ \pm}, 1 \frac{1}{2}^{ \pm}, 0 \frac{3}{2}^{ \pm}, 1 \frac{3}{2}^{ \pm}$are investigated using the QCD sum rule technique. Throughout the calculation, we emphasize the importance of the establishment of a valid Borel window, which corresponds to a region of the Borel mass, where the operator product expansion (OPE) converges and the presumed ground state pole dominates the sum rules. We obtain such a Borel window by constructing the sum rules from the differenece of two carefully chosen independent correlators and by calculating the OPE up to dimension 14 . As a result, we conclude that the state with qauntum numbers $0 \frac{3}{2}^{+}$state appears to be the most probable candidate for the experimantally observed $\Theta^{+}(1540)$, while we also obtain states with $0 \frac{1}{2}^{-}, 1 \frac{1}{2}^{-}, 1 \frac{3}{2}^{+}$at slightly higher mass regions. We furthermore discuss the contribution of the $K N$ scattering states to the sum rules, and the possible influence of these states on our results.
\end{abstract}

6th International Workshop on Chiral Dynamics, CD09

July 6-10, 2009

Bern, Switzerland

\footnotetext{
${ }^{*}$ Speaker.
} 


\section{Introduction}

The $\Theta^{+}(1540)$ resonance, carrying positive strangeness $(S=+1)$ and baryon number $(B=$ $+1)$, is clearly an exotic state, as its minimal quark content must be $u u d d \bar{s}$. After such a state was theoretically predicted by the chiral soliton model [1], it was first experimentally detected in 2003 by the LEPS group at SPring8 [2]. This has led to a large amount of experimental and theoretical works in the subsequent years, but many of the key problems surrounding $\Theta^{+}(1540)$, such as its unnaturally narrow width or its correct quantum number assignment, are still waiting to be solved.

Experimentally, the situation is rather unclear. After the CLAS collaboration has published several papers on their pentaquark search with high statistics [3, 4, 5, 6], where no signal of $\Theta^{+}(1540)$ could be found, many people now seem to believe that the pentaquark does not exist after all and that the whole story was just "a curious episode in the history of science" [7]. There are, however still experiments that still claim to observe a signal of $\Theta^{+}$[8, 9] and therefore this issue should not be considered to be completely settled yet. Additional experimental results, which either unambiguously confirm the existence of $\Theta^{+}(1540)$ or otherwise can eliminate it completely, are eagerly waited for.

The main subject of the present study is to compare the results of QCD sum rule calculations for various possible quantum numbers (isospin, spin and parity) of $\Theta^{+}(1540)$. From this comparison, we aim to determine which of the investigated quantum numbers is the one that most likely has to be assigned to the $\Theta^{+}(1540)$ state. Furthermore, we also look for possible excited states below $2 \mathrm{GeV}$, that can maybe found in future experiments. For these purposes we use an improved version of the QCD sum rule method, which has first been proposed in [10]. The basic idea of improvement is to use the difference of two correlators to construct the sum rule, which significantly suppresses the continuum part of the spectral function and therefore helps to find a valid Borel window, whose existence is a necessary condition for obtaining reliable results within the QCD sum rule technique. Moreover we calculate the OPE up to dimenstion 14, which is indispensable for a sufficient convergence of the expansion.

\section{Formalism}

In the QCD sum rule method [11, 12], the analytic properties of the two-point function

$$
\Pi(q)=i \int d^{4} x e^{i q x}\langle 0|T[\eta(x) \bar{\eta}(0)]| 0\rangle \equiv \Pi_{1}\left(q^{2}\right) \not q+\Pi_{2}\left(q^{2}\right)
$$

are used to extract information from the physical states that couple to the operator $\eta$. Here, $\Pi_{1}\left(q^{2}\right)$ is called the chiral-even, and $\Pi_{2}\left(q^{2}\right)$ the chiral-odd part. The analyticity of Eq.(2.1) allows one to write down the dispersion relation

$$
\Pi_{i}\left(q^{2}\right)=\frac{1}{\pi} \int_{0}^{\infty} d s \frac{\operatorname{Im}_{i}(s)}{s-q^{2}},
$$

for $i=1,2$.

In this study, we employ the usual "pole + continuum" parametrization for the spectral function, which appears in the imaginary part of the correlator:

$$
\operatorname{Im}_{i}(s)=\pi\left|\lambda_{i}\right|^{2} \delta\left(s-m_{\Theta^{+}}^{2}\right)+\theta\left(s-s_{t h}\right) \operatorname{Im} \Pi_{i}^{O P E}(s) .
$$


The $\delta$-function for the ground state pole is justified by the expereimental results, which show that the width of $\Theta^{+}(1540)$ is very narrow.

While the the low-energy part of the spectral function below the threshold parameter $s_{t h}$ is phenomenologically parametrized as in Eq.(2.3), the left hand side of Eq.(2.2) and the second term of Eq.(2.3) are calculated analytically using the operator product expansion (OPE). The results of this calculation for the chiral even part can be generally expressed as follows:

$$
\Pi_{1}^{O P E}\left(q^{2}\right)=\sum_{j=0}^{5} C_{2 j}\left(q^{2}\right)^{5-j} \log \left(-q^{2}\right)+\sum_{j=1}^{\infty} \frac{C_{10+2 j}}{\left(q^{2}\right)^{j}} .
$$

Here, $C_{i}$ contain various condensates and numerical factors.

Substituting Eq.(2.3) into the dispersion relation of Eq.(2.2), and applying the Borel transformation, the following expressions can be obtained (again for the chiral even part):

$$
\left|\lambda_{1}\right|^{2} e^{-m_{\Theta^{+}}^{2} / M^{2}}=-\int_{0}^{s_{t h}} d s e^{-s / M^{2}} \sum_{j=0}^{5} C_{2 j} s^{5-j}+\sum_{j=1}^{\infty} \frac{(-1)^{j} C_{10+2 j}}{\Gamma(j)\left(M^{2}\right)^{j-1}} \equiv f\left(M, s_{t h}\right) .
$$

From these equations, the expressions for $m_{\Theta^{+}}$and $\left|\lambda_{1}\right|^{2}$ can be exctracted straightforwardly:

$$
\begin{gathered}
m_{\Theta^{+}}^{2}\left(M, s_{t h}\right)=\frac{1}{f\left(M, s_{t h}\right)} \frac{\partial f\left(M, s_{t h}\right)}{\partial\left(-1 / M^{2}\right)}, \\
\left|\lambda_{1}\right|^{2}=f\left(M . s_{t h}\right) e^{m_{\Theta^{+}}^{2}\left(M, s_{t h}\right) / M^{2}} .
\end{gathered}
$$

Even though $m_{\Theta^{+}}\left(M, s_{t h}\right)$ in general depends on the Borel mass $M$ and the threshold parameter $s_{t h}$, this dependence should be weak in the case of a strong ground state pole dominating the spectral function below $s_{t h}$. On the other hand, if the $K N$ scattering states dominate the spectral function, a strong dependence of $m_{\Theta^{+}}\left(M, s_{t h}\right)$ on $M$ and $s_{t h}$ is expected.

\section{Importance of the Borel window}

To obtain reliable results with the QCD sum rule method, it is essential that the following two conditions

$$
\frac{L_{M}\left[\Pi_{\text {highest order terms }}^{O P E}\left(q^{2}\right)\right]}{L_{M}\left[\Pi_{\text {all terms }}^{O P E}\left(q^{2}\right)\right]} \leq 0.1
$$

( $L_{M}$ stands for the Borel transformation) and

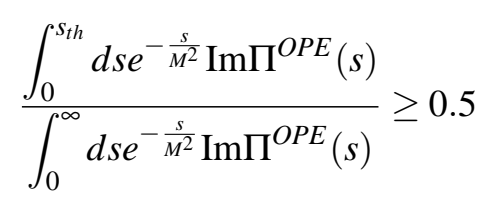

are satisfied for a certain region of the Borel mass, which is called the Borel window. Here, Eq.(3.1) has to be satisfied to make sure that the OPE converges sufficiently well, while Eq.(3.2) guarantees that the pole contribution dominates the sum rule.

However, in the case of most of the QCD sum rule calculations of pentaquark states so far, the two conditions $(3.1,3.2)$ have not been thoroughly checked, and no valid Borel window has 
been established [13]. The reason for this is firstly that the convergence of the OPE expansion for a correlator of an interpolating field containing five quarks is considerably slower than in the cases of interpolating fields containing only two or three quarks. Secondly, the high dimension of the interpolating field of a pentaquark causes the continuum part of the spectral function to be enhanced, which makes it difficult to obtain a high pole contribution.

A solution to this problem was proposed in a study by Kojo, Hayashigaki and Jido [10], where they made use of the chiral properties of two independent interpolating fields and considered, instead of one single correlator, the difference between two correlators of different interpolating fields. By this procedure, they realized a strong suppression of the leading orders of the OPE, which mainly contribute to the continuum part, and thus obtained a relatively large pole contribution.

We will follow the same lines of reasoning and consider two independent interpolating fields carrying the same quantum numbers $\eta_{1}$ and $\eta_{2}$, and then construct a more general operator by taking a linear combination of them:

$$
\eta(x)=\cos \theta \eta_{1}(x)+\sin \theta \eta_{2}(x)
$$

Defining the correlator calculated with this general interpolating field as $\Pi\left(q^{2}, \theta\right)$, we consider the difference of two independent correlators

$$
\Pi_{D}\left(q^{2}\right) \equiv \Pi\left(q^{2}, \theta_{1}\right)-\Pi\left(q^{2}, \theta_{2}\right)
$$

and constuct the sum rules for this new function $\Pi_{D}\left(q^{2}\right)$, for which we expect the leading orders of the OPE to be suppressed.

Finally, the values of the mixing angles $\theta_{1}$ and $\theta_{2}$ (in fact, the final result will only depend on the combination $\theta_{1}+\theta_{2}$ ) and the threshold parameter $s_{t h}$ have to be determined. To this end we emply the following to conditions:

1) A sufficiently wide Borel window exists.

2) $m_{\Theta^{+}}\left(M, s_{t h}\right)$ should only weakly depend on the Borel mass $M$ and on the threshold parameter $s_{t h}$.

We will thus choose those values of $\theta_{1}+\theta_{2}$ and $s_{t h}$ that most fully satify 1) and 2). Condition 1) is essential to obtain reliable results with the QCD sum rule method, while condition 2) corresponds to choosing the parameters so that the contribution of the $K N$ scattering states to the sum rule is as small as possible. This will discussed in more detail in the next section.

\section{Contribution of the $K N$ scattering states}

Generally, if $K N$ scattering states have the same quantum numbers as the interpolating fields, they may contribute to the sum rules to a certain extent. Therefore, we have to find a way to distinguish them from narrow pole states that we are really interested in. Let us first consider what the contribution of the $K N$ scattering states to the spectral function should look like. It is known that the $K N$ interaction is weak and slightly repulsive. We therefore just use phase space as a first 

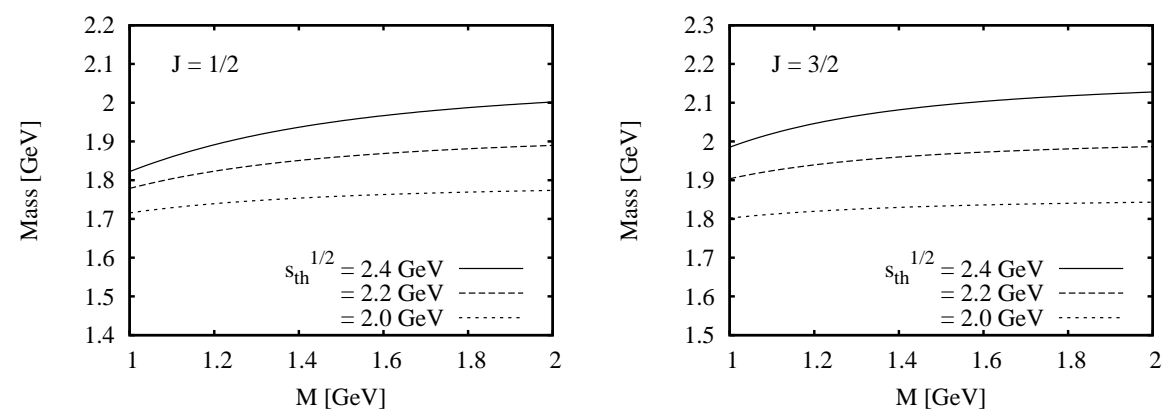

Figure 1: The "mass of the ground state pole" for spin $\frac{1}{2}$ and $\frac{3}{2}$, obtained when only the $K N$ scattering states contribute to the spectral function. Shown are the results of the chiral even part.

approximation of the $K N$ spectral function. This is a reasonable approximation as the qualitative results of this section do not depend on the detailed form of the spectral function.

We then compute the results that would be obtained by the QCD sum rules if only the $K N$ scattering states contribute to the spectral function. This means that we will calculate the quantity corresponding to Eq.(2.6), where for the spectral function we now use the expressions obtained from phase space. The results of the chiral even part for spin $\frac{1}{2}$ and spin $\frac{3}{2}$ are given in Fig. 1 .

It is clearly seen that while the dependence on the Borel mass $M$ is relatively weak, the results depend strongly on the threshold parameter $s_{t h}$. This can intuitively be understood from the fact that the spectral function containing only the phase space contribution is a fastly growing function when the energy is increased. Therefore, the high energy regions near the threshold parameter $s_{t h}$ will dominate the sum rule, which then leads to a behaviour as seen in Fig. 1 ,

The results of this section show that the dependence of $m_{\Theta^{+}}^{2}\left(M, s_{t h}\right)$ on $s_{t h}$ provides us with an indicator of how much the $K N$ scattering states contribute to the sum rule: a linear dependence of the same order as in Fig. 1 suggests a large contribution of the scattering states, while a significantly smaller dependence indicates that a narrow pole exists and is the dominant structure in the spectral function.

\section{Results}

The results of the sum rules of the chiral even part for the various quantum numbers are given in Fig. 2. These plots show the position of the presumed ground state pole $m_{\Theta^{+}}\left(M, s_{t h}\right)$ (see Eq. (2.6)) as a function of the Borel mass $M$ for three different values of the threshold parameter $s_{t} h$. The arrows indicate the boundaries of the Borel window. It is seen that we are able to obtain a valid Borel window for the sum rules of all the investigated quantum numbers. Furthermore, it has to be noted that the results of the chiral even part contain contributions from both positive and negative parity states. It is thus necessary to, for instance, perform the parity projected sum rules [14] to determine the parity of the states under investigation. That is what we have done, but the detailed results of these calculations will have to be presented elsewhere [15]. We here just state the final conclusions, which are identical for isospin $I=0$ and $I=1$ : in the spin $\frac{1}{2}$ case we have found that the plots shown in Fig. 2 are dominated by negative parity states, while the parity for the spin $\frac{3}{2}$ 

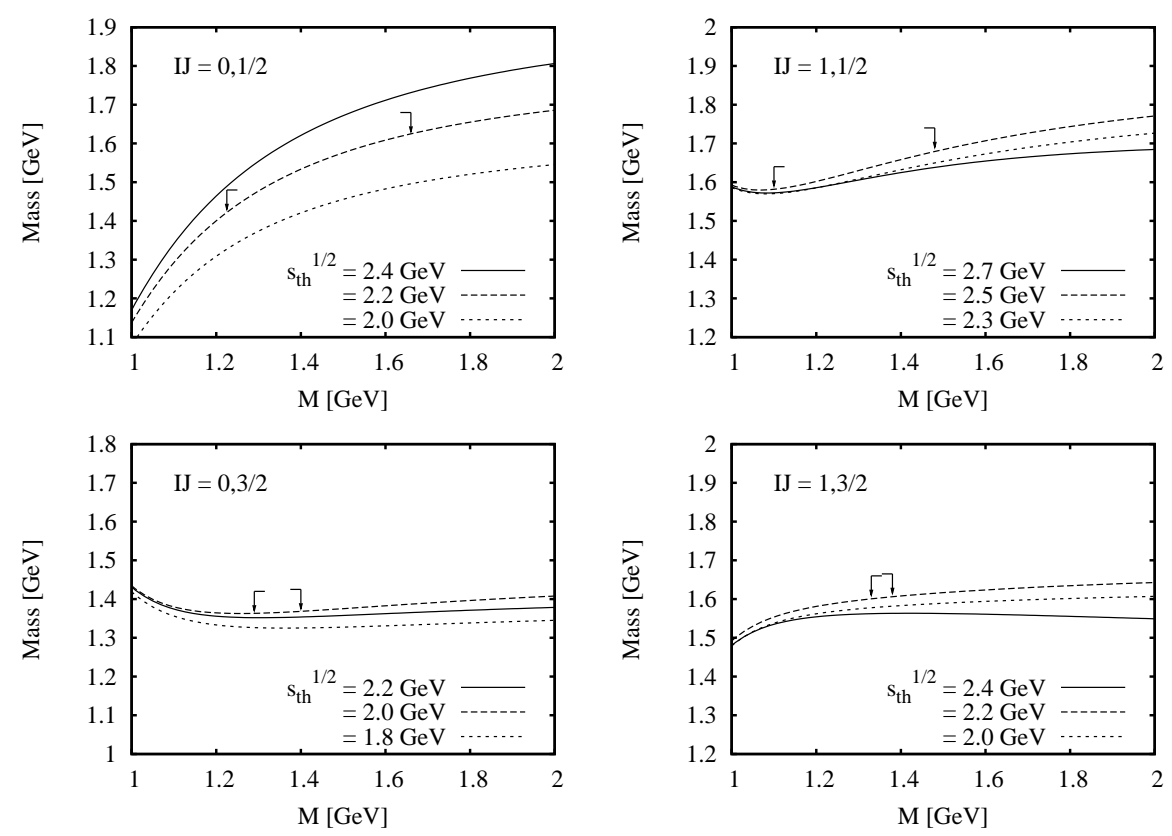

Figure 2: The mass of the pentaquark for several quantum number as a function of the Borel mass $M$. The arrows indicate the boundary of the Borel window for the middle value of $\sqrt{s_{t h}}$. The results are obtained from the chiral even part.

states turned out to be positive. Putting everything together, these results can be summarized as in Table 1 .

The statement "no state found below $2.0 \mathrm{GeV}$ " in Table 1 means that either no valid Borel window could be found or that the results of the sum rules did strongly depend on $M$ and $s_{t h}$ and that therefore no evidence for a narrow ground state pole could be found. As is seen in Fig. 2, the result for $I L^{P}=0 \frac{1}{2}^{ \pm}$depends on $M$ and $s_{t h}$ quite strongly, which suggests that we are here probably observing mainly $K N$ scattering states. After parity projecting this result to negative parity, this dependence on $M$ and $s_{t h}$ becomes in fact much weaker, which may signify that the $K N$ scattering

Table 1: Summarized results for all quantum numbers that have been investigated. The allowed $K N$ decay channels of the respective quantum numbers are indicated in brackets.

\begin{tabular}{|c|c|c|c|}
\hline \multicolumn{2}{|c|}{} & \multicolumn{2}{c|}{ Parity } \\
\cline { 3 - 4 }$J=\frac{1}{2}$ & $I=0$ & $\begin{array}{c}\text { no state found below } 2.0 \mathrm{GeV} \\
(K N \text { P-wave) }\end{array}$ & $\begin{array}{c}1.5 \pm 0.3 \mathrm{GeV}(?) \\
(K N \text { S-wave) }\end{array}$ \\
\cline { 2 - 4 } & $I=1$ & $\begin{array}{c}\text { no state found below } 2.0 \mathrm{GeV} \\
(K N \text { P-wave) }\end{array}$ & $\begin{array}{c}1.6 \pm 0.4 \mathrm{GeV} \\
(K N \text { S-wave) }\end{array}$ \\
\hline \multirow{2}{*}{$J=\frac{3}{2}$} & $I=0$ & $\begin{array}{c}1.4 \pm 0.2 \mathrm{GeV} \\
(K N \text { P-wave) }\end{array}$ & $\begin{array}{c}\text { no state found below } 2.0 \mathrm{GeV} \\
\text { (KN D-wave) }\end{array}$ \\
\cline { 2 - 4 } & $I=1$ & $\begin{array}{c}1.6 \pm 0.3 \mathrm{GeV} \\
(K N \text { P-wave) }\end{array}$ & $\begin{array}{c}\text { no state found below } 2.0 \mathrm{GeV} \\
\text { (KN D-wave) }\end{array}$ \\
\hline
\end{tabular}


states with positive parity are contaminating the chiral even part and therefore a result such as in Fig. 2 is obtained. Nevertheless, concerning this state we can not be completely conclusive and therefore have to put a question mark behind this result.

Next, we discuss the physical implications of the obtained results. A question that comes to ones mind when looking at Table 1 is, why can we not observe $J^{\pi}=\frac{1}{2}^{+}$states while we are seeing the ones with $J^{\pi}=\frac{3}{2}^{+}$? These states are in some models considered to be spin-orbit partners [16], so if these models are realistic and constitent with QCD, we should be able to observe both of these states. There are at least two possible explanations for our results. One explanation could be that the states with $J^{\pi}=\frac{1}{2}^{+}$in fact exist, but their coupling to the used operators are too small and/or the $K N$ scattering contribution is too large, so that a narrow peak structure cannot be extracted. Another possible interpretation of the missing $J^{\pi}=\frac{1}{2}^{+}$states could be that, the spin-orbit partners of the spin $\frac{3}{2}$ states are not the ones with spin $\frac{1}{2}$ but with spin $\frac{5}{2}$. This would mean that $\Theta^{+}$is indeed a very exotic state, as in this case the uudd quarks have to form a spin 2 state, which would then couple to the remaining $\bar{s}$. This is of course only a very speculative conjecture, but it would be interesting to test it by calculating pentaquark states with spin $\frac{5}{2}$.

Another important point, that needs to be discussed, is the interpretation of the observed $J^{\pi}=$ $\frac{1}{2}^{-}$states. This state was also found in a lattice study (conducted only for the isosinglet state), where a resonance state was isolated from the $K N$ scattering states [17]. Our results (especieally in the isosinglet case) are somewhat ambiguous, and the error bars are large, so it is difficult to draw any definite conclusions. In any case, whether such states turn out to be real pentaquark resonances or not, they most possibly do not correspond to the observed $\Theta^{+}$state, because $J^{\pi}=\frac{1}{2}^{-}$states can decay into $K N$ by an $\mathrm{S}$-wave, for which the width is expected to be much larger than the observed value for $\Theta^{+}$, which is even less than $1 \mathrm{MeV}$ [8]. Of course, in principle there may exist some so far unknown mechanism, which suppresses the width strongly and which would allow to assign the $J^{\pi}=\frac{1}{2}^{-}$quantum numbers to the $\Theta^{+}(1540)$, but with our present knowledge and experience, this seems to be unlikely.

\section{Conclusion}

We have studied the $S=+1$ pentaquark states with quantum numbers $I J^{\pi}=0 \frac{1}{2}^{ \pm}, 1 \frac{1}{2}^{ \pm}, 0 \frac{3}{2}^{ \pm}, 1 \frac{3}{2}^{ \pm}$, to see which one is the most likely candidate for the observed $\Theta^{+}(1540)$ state. To do this, we have employed the QCD sum rule method, whose reliability is improved by analysing the difference of two independent correlators, by which the contribution of the high-energy continuum states is suppressed. Furthermore, by calculating the OPE up to dimension 14 it is made sure that the expansion is converging well, and a valid Borel window can therefore be established.

The results for each quantum number are given in Table 1 . Considering first the spin $\frac{1}{2}$ states, we could observe some evidence for resonance states with $I J^{\pi}=0 \frac{1}{2}^{-}$and $1 \frac{1}{2}^{-}$in the region of $1.5 \mathrm{GeV}$. As discussed in the previous section, we do not believe that these states correspond to $\Theta^{+}(1540)$, because their width is expected to be too large to be consistent with the experimental value.

On the other hand, we have also found evidence for resonance states with $I J^{\pi}=0 \frac{3}{2}^{+}$and $1 \frac{3}{2}^{+}$. In both of these cases the values of the masses show only a weak dependence on the Borel mass $M$ and the threshold parameter $s_{t h}$, as can be confirmed from Fig. 2. For the isosinglet case this 
was already pointed out in [18]. This suggests that we are really observing narrow resonance states in the spectral functions of these quantum numbers. As no isospin partners of the $\Theta^{+}$have so far been found, it is believed to be an isosinglet. We therefore conclude from our results that the most probable quantum number candidate for $\Theta^{+}(1540)$ is $I J^{\pi}=0 \frac{3}{2}^{+}$.

\section{Acknowledgments}

This work was partially supported by KAKENHI, 17070002 (Priority area), 19540275 and 20028004. A part of this work was done in the Yukawa International Project for Quark-Hadron Sciences (YIPQS). P.G. acknowledges the support by the Japan Society for the Promotion of Science for Young Scientists and is thankful for the hospitality of the Yukawa Institute for Theoretical Physics at Kyoto University, where part of this work has been completed. T.K. is supported by RIKEN, Brookhaven National Laboratory and the U. S. Department of Energy [Contract No. DEAC02-98CH10886].

\section{References}

[1] D. Diakonov, V. Petrov, and M. Polyakov, Z. Phys. A 359, 305 (1997).

[2] T. Nakano et al. (LEPS Collaboration), Phys. Rev. Lett. 91, 012002 (2003).

[3] M. Battaglieri et al. (CLAS Collaboration), Phys. Rev. Lett. 96, 042001 (2006).

[4] B. McKinnon et al. (CLAS Collaboration), Phys. Rev. Lett. 96, 212001 (2006).

[5] S. Niccolai et al. (CLAS Collaboration), Phys. Rev. Lett. 97, 032001 (2006).

[6] R. De Vita et al. (CLAS Collaboration), Phys. Rev. D 74, 032001 (2006).

[7] C. Amsler et al. [Particle Data Group], Phys. Lett. B 667, 1 (2008).

[8] V.V. Barmin et al. (DIANA Collaboration), Phys. Atom. Nucl. 70, 35 (2007).

[9] T. Nakano et al. (LEPS Collaboration), Phys. Rev. C 79, 025210 (2009).

[10] T. Kojo, A. Hayashigaki, and D. Jido, Phys. Rev. C 74, 045206 (2006).

[11] M.A. Shifman, A.I. Vainshtein, and V.I. Zakharov, Nucl. Phys. B147, 385 (1979); B147, 448 (1979).

[12] L.J. Reinders, H. Rubinstein, and S. Yazaki, Phys. Rep. 127, 1 (1985).

[13] R.D. Matheus and S. Narison, Nucl. Phys. (Proc. Suppl.) B152, 236 (2006).

[14] D. Jido, N. Kodama, and M. Oka, Phys. Rev. D 54, 4532 (1996).

[15] P. Gubler, D. Jido, T. Kojo, T. Nishikawa, and M. Oka, in preperation.

[16] R. Jaffe and F. Wilczek, Phys. Rev. Lett. 91, 232003 (2003).

[17] T.T. Takahashi, T. Umeda, T. Onogi, and T. Kunihiro, Phys. Rev. D 71, 114509 (2005).

[18] P. Gubler, D. Jido, T. Kojo, T. Nishikawa, and M. Oka Phys. Rev. D 79, 114011 (2009). 